\title{
Quantization of the superconducting energy gap in an intense microwave field
}

\author{
A. A. Boris and V. M. Krasnov* \\ Department of Physics, Stockholm University, AlbaNova University Center, SE-10691 Stockholm, Sweden
}

(Dated: September 25, 2021)

\begin{abstract}
We study photon assisted tunneling in $\mathrm{Nb} / \mathrm{AlO}_{x} / \mathrm{Nb}$ Josephson junctions. A quantitative calibration of the microwave field in the junction allowed direct verification of the quantum efficiency of microwave photon detection by the junctions. We observe that voltages of photon assisted tunneling steps vary both with the microwave power and the tunneling current. However, this variation is not monotonous, but staircase-like. The phenomenon is caused by mutual locking of positive and negative step series. A similar locking is observed with Shapiro steps. As a result the superconducting gap assumes quantized values equal to multiples of the quarter of the photon energy. The quantization is a manifestation of nonequilibrium tuning (suppression or enhancement) of superconductivity by the microwave field.
\end{abstract}

Superconducting Josephson junctions are used as sensitive detectors of microwave (MW) and terahertz signals [1]. Application of MW radiation leads to appearance of Shapiro and photon assisted tunneling (PAT) steps in the current-voltage $(I-V)$ characteristics of a junction at $e V= \pm n h f / 2$ and $e V= \pm 2 \Delta \pm n h f$, respectively. Here $f$ is the MW frequency and $\Delta$ is the superconducting energy gap. Shapiro and PAT steps originate from Cooper pair and quasiparticle (QP) currents, correspondingly (for details see e.g. Ref. 9]). A response of junctions to weak MW signals is well understood [1]3]. For example, the differential conductivity due to PAT is described by the Tien-Gordon theory:

$$
\frac{d I}{d V}(V)=\sum_{n=-\infty}^{\infty} J_{n}^{2}\left(\frac{e V_{M W}}{h f}\right) \frac{d I_{0}}{d V}\left(V+\frac{n h f}{e}\right),
$$

where $J_{n}$ are Bessel functions of integer order $n, V_{M W}$ is the MW voltage amplitude in the junction and $I_{0}(V)$ is a dc-current without MW. Positive/negative $n$ terms in this expression describe contributions from PAT with absorbtion/emission of $n$ photons.

However, such a textbook description works only for weak MW signals that do not affect superconducting properties of junction electrodes. Strong electromagnetic fields may disturb a thermal equilibrium state. This may either suppress [10, 11] or enhance [12 15] superconducting properties, such as $\Delta$ and the critical current $I_{c}$. Current flow through the junctions also leads to disruption of the equilibrium. Analysis of such nonequilibrium effects at low temperatures is complicated by essentially nonlinear response of the junction to perturbations [16]. Detailed understanding of nonequilibrium effects in Josephson junctions is lacking and is important both for optimization of operation of superconducting quantum devices [17 22] and for fundamental studies of the pairing mechanism in unconventional superconductors [23]. To investigate the influence of nonequilibrium effects in intense MW fields $V_{M V}>2 \Delta / e$ on detection characteristics of Josephson junctions is the main objective of our work.
In this work we study photon assisted tunneling steps in $\mathrm{Nb} / \mathrm{AlOx} / \mathrm{Nb}$ Josephson junctions. Using an absolute calibration of the MW field inside the junction we demonstrate quantum efficiency of microwave photon detection by our junctions. We observe that contrary to expectations PAT voltages are not constant, but vary both with the MW power $P_{M W}$ and the current through the junction. However, this variation is not monotonous, but staircase-like. The phenomenon is caused by mutual locking of positive $e V=2 \Delta-n h f$ and negative $e V=-2 \Delta+m h f$ PAT series at large MW amplitudes $e V_{M W} \gtrsim \Delta$. A similar locking is observed between Shapiro and PAT steps. The locking is a manifestation of nonequilibrium adjustment of the superconducting gap. As a result, $\Delta$ assumes quantized values equal to multiples of the quarter of the photon energy.

We study micron-size $\mathrm{Nb} / \mathrm{AlO}_{x} / \mathrm{Nb}$ junctions made by a standard technology. Several junctions with different sizes on the same chip are studied. Detailed characterization of our junctions can be found in Ref. 24. Measurements are performed in a pulsed-tube cooled optical cryostat. A single loop frequency synthesizer with a 8x multiplier is used as a MW source. The MW power is supplied to the junction quasioptically using a system of high-density polyethylene lenses. The MW power $P_{M W}$ is tuned using two polarizers. The polarizer at the source site is rotated by a step motor. The polarizer at the sample site is aligned with the source and is fixed. The applied MW power is measured using an opto-acoustical detector (Golay cell).

Figure 1 (a) shows $I-V$ characteristics of a junction at zero magnetic field for several $P_{M W}$ at $f \simeq 76.6 \mathrm{GHz}$. With increasing $P_{M W}$ PAT steps at $e V= \pm 2 \Delta \pm n h f$ and Shapiro steps at $e V= \pm n h f / 2$ appear. Step amplitudes oscillate with $P_{M W}$. The supercurrent can be suppressed by applying magnetic field parallel to the junction plane, corresponding to integer number of flux quanta $\Phi_{0}$ in the junction. In this case only PAT steps are present. Fig. 1. (b) represents $d I / d V(V)$ curves, normalized by the tunnel resistance $R \simeq 3.6 \Omega$, at $\Phi=2 \Phi_{0}$ for a slightly smaller junction. Curves are presented for MW powers 

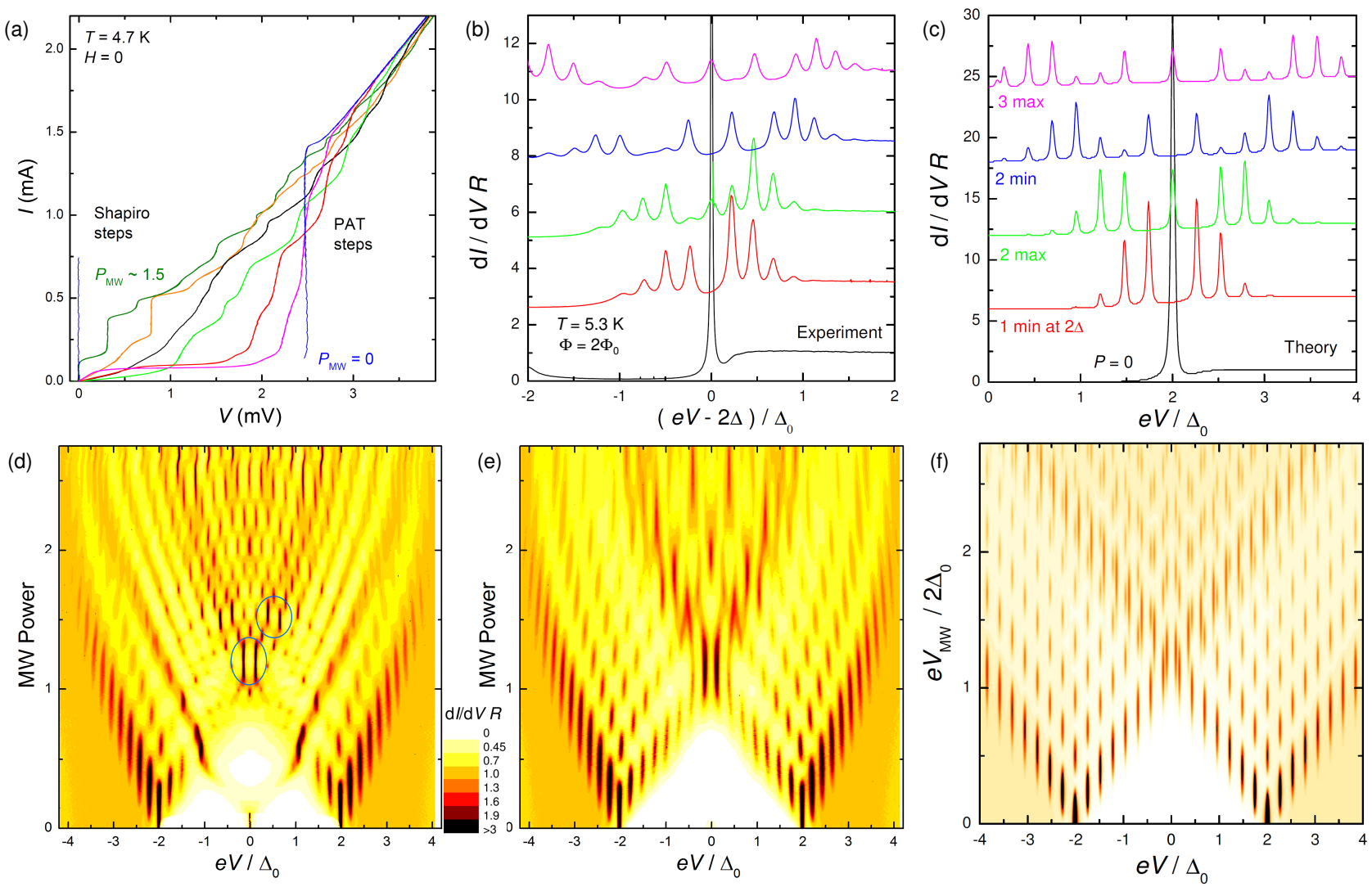

FIG. 1. (Color online). (a) Current-voltage characteristics at $H=0$ and $T=4.7 \mathrm{~K}$ for different $P_{M W}=0,0.15,0.275,0.463$, $0.825,1.25,1.5$ at $f=76.6 \mathrm{GHz}$. (b) Experimental and (c) theoretical $d I / d V(V)$ curves with PAT steps for several $P_{M W}$ corresponding to maxima and minima of the sum-gap step $e V=2 \Delta$. Curves are offset vertically for clarity. (d) and (e) Color maps of differential conductances for the same junction at $T=5.3 \mathrm{~K}$ for (d) $H=0$ and (e) $\Phi=2 \Phi_{0}$. Both Shapiro and PAT steps are seen in (d), but only PAT steps are present in (e). (f) Theoretical color map of $d I / d V$ for PAT as a function of bias voltage $V$ and the MW voltage inside the junction $V_{M W}$. Note that positive and negative step series overlap at $e V_{M W} / 2 \Delta_{0} \simeq 1$.

corresponding to maxima and minima of the sum-gap step $\mathrm{eV}=2 \Delta$. In Fig. 11(c) we show corresponding theoretical calculations according to Eq. (1) 25] (curves for different $P_{M W}$ in Figs. 1 (b) and (c) are offset vertically for clarity).

Figs. 1(d) and (e) represent color maps of $d I / d V R$ as a function of bias voltage and MW power. Measurements are performed at $T=5.3 \mathrm{~K}$ for (d) $H=0$ and (e) at $\Phi=2 \Phi_{0}$. At $H=0$ both Shapiro and PAT steps are present. At $\Phi=2 \Phi_{0}$ solely the PAT step structure is seen. Fig. 1(f) shows a corresponding theoretical calculation for PAT, obtained from Eq. (1), as a function of the MW voltage. It is seen that with increasing $V_{M W}$ two PAT step series start to expand in a V-shape manner from the sum-gap peaks at positive $e V=2 \Delta \pm n h f$ and negative $e V=-2 \Delta \pm m h f$ voltages. The steps modulate quasi-periodically with increasing $V_{M W}$. At $e V_{M W}=2 \Delta$ photon absorption steps from the two series overlap. We use this point for absolute calibration of the MW amplitude inside the junction. In what follows we normalize $P_{M W}$ to this value.
Comparison of theory with experiment reveals a qualitative agreement, but also certain differences. First, in theory PAT steps oscillate quasi-periodically with $V_{M W}$, while in experiment with $P_{M W}$ [26. This may indicate that we have reached the quantum efficiency of photon detection [1], in which case the number of tunneled electron is limited by the number of incoming photons. The quantum-limited current at the $n$-th photon absorption step is $I_{n}=(e / n) d N / d t$, where $d N / d t$ is the rate of incoming photons, connected to the absorbed MW power $P_{a}=h f d N / d t$. Thus, such current is proportional to the absorbed MW power rather than $V_{M W}, I_{n}=P_{a} e / n h f$. Usually, however, it is difficult to identify exactly how much MW power is absorbed by the junction. Therefore, to verify this idea we propose to use the absolute calibration of the MW field inside the junction at the overlap point $V_{M W}\left(P_{M W}=1\right) \simeq 2 \Delta / e$, which yields $P_{a}=V_{M W}^{2} / 4 R=P_{M W}\left(\Delta^{2} / e^{2} R\right)$. Here an additional factor $1 / 2$ is due to rectifying action of the junction due to which at positive dc-bias the MW current is flowing only during positive half-periods of oscillations 

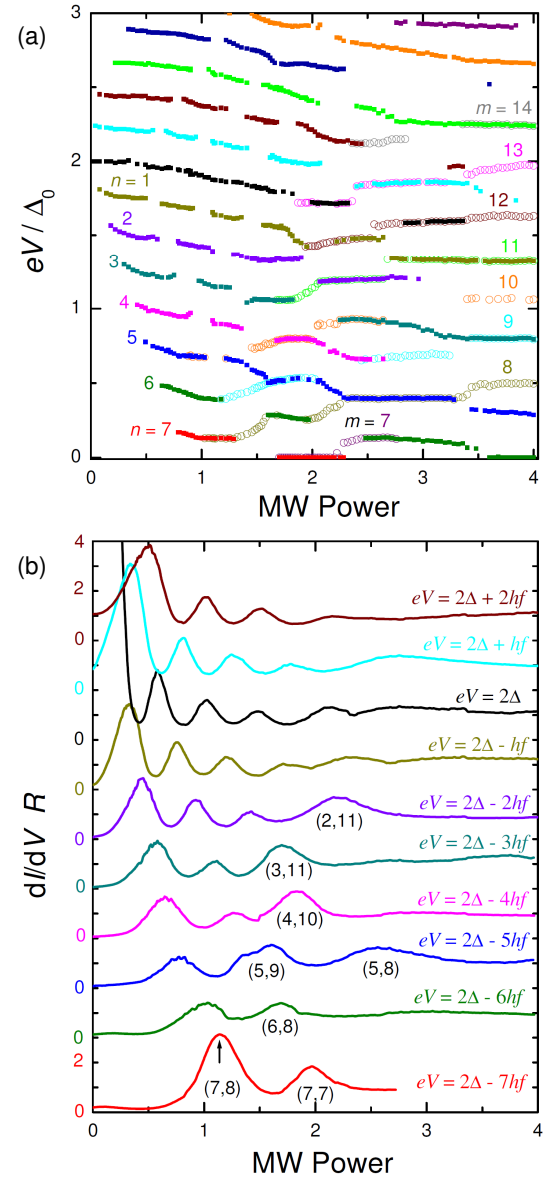

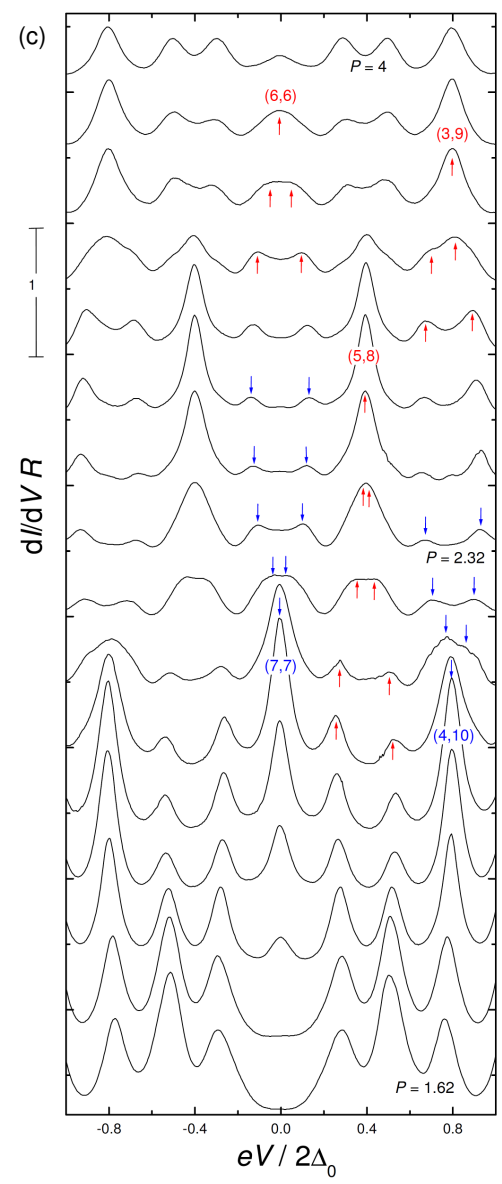

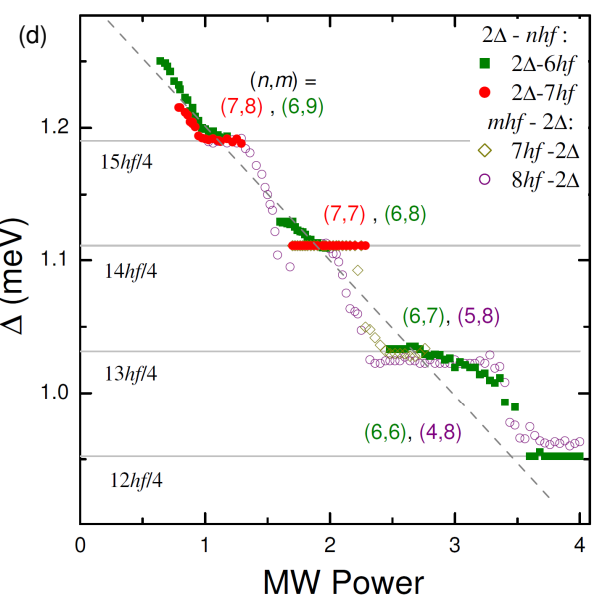

(e) Photon absorption processes

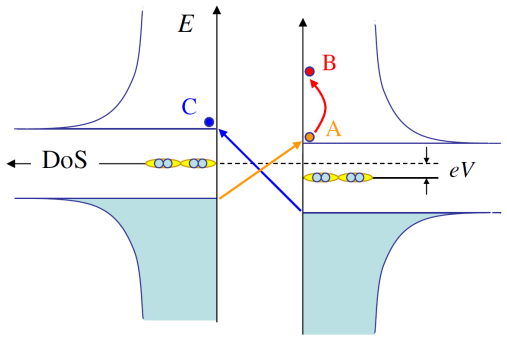

A: Positive PAT step $e V=2 \Delta-n h f$ B: Up-scattering of a QP

C: Negative PAT step $e V=-2 \Delta+m h f$

FIG. 2. (Color online). (a) PAT step voltages versus MW power from Fig. 1 (c). Solid and open symbols represent positive and negative PAT step series with orders $n$ and $m$, respectively. Note locking of those steps upon overlap. (b) Normalized step amplitude as a function of MW power. Numbers $(n, m)$ indicate formation of high amplitude steps due to locking of the corresponding positive $n$ and negative $m$ steps. (c) $d I / d V(V)$ curves at $P_{M W}$ from 1.62 to 4 . Up/down arrows indicate merging/splitting of PAT steps, leading to appearance/dissapearance of high amplitude steps. Curves in (b) and (c) are shifted vertically for clarity. (d) The superconducting energy for several PAT steps. Quantization of the gap at $P_{M W}>1$ is seen. (e) Diagrams of various nonequilibrium processes, associated with photon absorption.

and $P_{M W}$ is the normalized MW power to the overlap point value. Thus, quantum-limited $n$-photon current is $I_{n}=P_{M W}(\Delta / n h f)(\Delta / e R)$. For the case of Fig. 1 (a), $\Delta \simeq 2.5 \mathrm{mV}, \Delta / h f \simeq 3.9$ and the sum-gap kink amplitude $2 \Delta / e R \simeq 1.4 \mathrm{~mA}$, we get $I_{n} \simeq(2.7 / n) P_{M W}(\mathrm{~mA})$. Magenta line in Fig. 1 (a), $P_{M W} \simeq 0.15$, corresponds to the first maximum of the single photon step, at which contribution from multi-photon tunneling is small. It is seen that the amplitude of the single photon absorption step, $e V=2 \Delta-h f$, is very close to the calculated $I_{1} \simeq 0.4 \mathrm{~mA}$, confirming the quantum efficiency of detection of MW photons by our junctions.

Another difference between experiment and theory, which is in focus of this work, occurs at high MW powers $P_{M W}>1$ when positive and negative PAT steps overlap. In theory, Fig. 1 (f), this does not lead to anything special. But in experiment, Fig. 1 (e), it clearly causes a complete reconstruction of the step pattern. Instead of having two independent series of steps, the nearby steps from positive and negative sides lock together forming fewer steps with significantly stronger amplitudes. A similar locking effect is seen in Fig. 1(d) between Shapiro and PAT steps, which leads to appearance of super-steps, marked by ovals. Such super-steps do not occur in the absence of PAT [8].

Figure 2 (a) shows PAT step voltages from Fig. 11 (e), normalized by the gap $\Delta_{0}$ at $P_{M W}=0$. It is seen that contrary to a textbook case step voltages are neither constant nor equidistant. Since PAT voltages are strictly connected to the gap, $e V_{n}= \pm 2 \Delta \pm n h f$, variation of step voltages implies variation of $\Delta$. Generally, step voltages decrease with increasing $P_{M W}$. This indicates suppression of the gap by the MW field. We emphasize that the base temperature was actively controlled during the experiment and was constant in the whole range of $P_{M W}$ with the accuracy better than $0.1 \mathrm{~K}$. Thus, suppression of 
$\Delta$ is due to nonequilibrium over-population of the electronic system [16, rather than overheating of the system. Suppression of $\Delta$ leads to a decrease of positive $e V_{n}=2 \Delta-n h f$ (solid symbols) and increase of negative $e V_{m}=-2 \Delta+m h f$ (open symbols) photon absorption steps. Thus, positive and negative steps gradually approach each other and overlap at $P_{M W}>1$. As seen from Fig. 1 (e), the overlap leads to a complete reconstruction of the PAT pattern at $P_{M W}>1$ : nearby positive and negative steps lock-in together to form high-amplitude steps with constant (power-independent) voltages.

In Fig. 2 (b) we show amplitudes of PAT steps from Fig. 2 (a) of different $n$ as a function of the MW power (symbols and lines with the same color in Figs. 2 (a) and (b) represent steps of the same order). Periodic modulation is clearly seen (see also the Supplementary [26]). We mark steps formed by locking of $n$-th order positive and $m$-th order negative photon absorption steps by doublets $(n, m)$. They have significantly higher amplitudes than foregoing maxima with the same $n$, or than corresponding maxima of the proceeding order $n-1$. For example the third maximum for $n=3$, marked $(3,11)$, is due to locking between $n=3$ and $m=11$ steps. It has a larger amplitude than both the second $n=3$ and the third $n=2$ maxima. Fig. 2 (c) shows evolution of $d I / d V(V)$ curves in the range of $P_{M W}$ from 1.62 to 4. Up/down arrows indicate merging/splitting of PAT steps, leading to appearance/dissapearance of high amplitude steps $(n, m)$.

A condition for locking of positive and negative photon absorption steps is $2 \Delta-n h f=m h f-2 \Delta$. Therefore, upon such locking the gap assumes quantized values,

$$
\Delta=(n+m) h f / 4 .
$$

Fig. 2 (d) shows the energy gap vs. MW power for several PAT steps. The gap is obtained explicitly as $\Delta=$ $\left(e V_{n}+n h f\right) / 2$ for positive and $\Delta=\left(m h f-e V_{m}\right) / 2$ negative step series. A staircase-like variation of $\Delta\left(P_{M W}\right)$ with platos at quarters of the photon energy is clearly seen. The quantization becomes progressively stronger with increasing the MW power. Ovals in Fig. 11(d) indicate that a similar locking takes place between Shapiro and PAT steps. This occurs when $n h f / 2=2 \Delta-m h f$ leading to the same type of quantization, Eq. (2). Locking between Shapiro and PAT steps suggests that the locking involves collective ac-Josephson currents both for pairs and quasiparticles, such as the illusive QP interference current (the cosine term) 9].

Finally we want to discuss the mechanism of the observed gap quantization. Fig. 2 (e) represents diagrams of three possible photon absorption processes. The process A corresponds to positive PAT steps. It leads to breaking of a Cooper pair and appearance of nonequilibrium electron and hole-like QPs, which suppresses $\Delta$. The process $\mathrm{B}$ corresponds to up-scattering of a quasiparticle. According the the self-consistency equation, $\Delta$ is most sensitive to QPs just at the bottom of the gap (see e.g. Ref. [16]). Therefore the process B leads to enhancement of the gap. The process $\mathrm{C}$ corresponds to negative PAT steps. On one hand, it leads to enhanced nonequilibrium QP population and thus decreases $\Delta$, on the other hand the current is flowing against the voltage, leading to negative dissipation power and cooling of the junction, which enhances $\Delta$. Thus there are several nonequilibrium processes in the MW field that may either increase or decrease $\Delta$. Such a tunability is required for locking of PAT steps. The dashed line in Fig. 2 (d) indicates that the gap in average is decreasing approximately linearly with increasing $P_{M W}$. However at the steps it deviates both up and down from this line showing that both enhancement and suppression of the gap indeed takes place.

To conclude, quantitative calibration of the microwave field in Josephson junction allowed direct verification of the quantum efficiency of microwave photon detection by the junctions. Our main result is observation of a quantization of the superconducting gap in intense microwave fields. The phenomenon is caused by mutual locking of photon assisted tunneling steps due to nonequilibrium enhancement and suppression of superconductivity by the microwave field.

The work was supported by the Swedish Foundation for International Cooperation in Research and Higher Education Grant No. IG2013-5453 and the Swedish Research Council Grant No. 621-2014-4314.

* Vladimir.Krasnov@fysik.su.se

[1] J.R. Tucker and M. J. Feldman, Rev. Mod. Phys. 57, 1055 (1985).

[2] I. A. Devyatov, L. S. Kuzmin, K. K. Likharev, V.V. Migulin, and A.B. Zorin, J. Appl. Phys. 60, 1808 (1986).

[3] Q. Hu, C.A. Mears, P.L. Richards, and F.L. Lloyd, Phys. Rev. B 42, 10250 (1990).

[4] Yu. P. Gousev, G. N. Gol'tsman, B. S. Karasik, E. M. Gershenzon, A. D. Semenov, H. S. Barowski, R. S. Nebosis, and K. E Renk, Int. J. IRMMW 17, 317 (1996).

[5] V. P. Koshelets and S. Shitov, Supercond. Sci. Technol. 13, R53 (2000).

[6] Y. Y. Divin, U. Poppe, O. Y. Volkov and V. V. Pavlovskii, Appl. Phys. Lett. 76, 2826 (2000).

[7] J. Basset, H. Bouchiat, and R. Deblock, Phys. Rev. B 85, 085435 (2012).

[8] M. Malnou, C. Feuillet-Palma, C. Ulysse, G. Faini, P. Febvre, M. Sirena, L. Olanier, J. Lesueur, and N. Bergeal, J. Appl. Phys. 116, 074505 (2014).

[9] A. Barone and C. Paterno, Physics and Applications of the Josephson effect (J. Willey \& Sons, New York 1982).

[10] D. Winkler and T. Claeson, J. Appl. Phys. 82, 4482 (1987).

[11] E. M. Gershenzon, G.N. Gol'tsman, V.D. Potapov and A.V. Sergeev, Sol. St. Commun. 75, 639 (1990).

[12] A.F.G. Wyatt, V.M. Dmitriev, W.S. Moore, and F.W. 
Sheard, Phys. Rev. Lett. 16, 1166 (1966).

[13] T. Kommers and J. Clarke, Phys. Rev. Lett. 38, 1091 (1977).

[14] R.E. Horstman and J. Wolter, Phys. Lett. 82A, 43 (1981).

[15] M. Beck, I. Rousseau, M. Klammer, P. Leiderer, M. Mittendorff, S. Winnerl, M. Helm, G. N. Gol'tsman, and J. Demsar, Phys. Rev. Lett. 110, 267003 (2013).

[16] V.M. Krasnov, Phys. Rev. Lett. 103, 227002 (2009).

[17] J. Wenner, et al., Phys. Rev. Lett. 110, 150502 (2013).

[18] V.F. Maisi, S.V. Lotkhov, A. Kemppinen, A. Heimes, J.T. Muhonen, and J.P. Pekola Phys. Rev. Lett. 111, 147001 (2013).

[19] S.E. de Graaf, J. Leppäkangas, A. Adamyan, A.V. Danilov, T. Lindström, M. Fogelström, T. Bauch, G. Johansson, and S.E. Kubatkin, Phys. Rev. Lett. 111, 137002 (2013).

[20] P. J. de Visser, D. J. Goldie, P. Diener, S. Withington, J. J. A. Baselmans, and T. M. Klapwijk, Phys. Rev. Lett. 112, 047004 (2014).

[21] U. Vool, I. M. Pop, K. Sliwa, B. Abdo, C. Wang, T. Brecht, Y. Y. Gao, S. Shankar, M. Hatridge, G. Catelani,
M. Mirrahimi, L. Frunzio, R. J. Schoelkopf, L. I. Glazman, and M. H. Devoret, Phys. Rev. Lett. 113, 247001 (2014).

[22] A. Pourkabirian, M. V. Gustafsson, G. Johansson, J. Clarke, and P. Delsing, Phys. Rev. Lett. 113, 256801 (2014).

[23] V. M. Krasnov, S.O. Katterwe and A. Rydh, Nature Commun. 4, 2970 doi: 10.1038/ncomms3970 (2013).

[24] V. M. Krasnov, H. Motzkau, T. Golod, A. Rydh, S. O. Katterwe and A. B. Kulakov Phys. Rev. B 84, 054516 (2011).

[25] Calculations according to Eq. (1) neglect possible variation of $V_{M V}(V)$ due to quantum susceptance contribution [1, 3]. We did perform additional calculations taking into account the effect of quantum susceptance. It may lead to appearance of negative conductance, but does not lead to mutual locking of the steps and, therefore, does not introduce significant changes into the pattern.

[26] See supplementary material at http://link.aps.org/supplemental/XX.XXX/PhysRevLett.XXX.XXX 